\title{
The Strong Transformation of Spiral Galaxies Infalling into Massive Clusters at $\mathrm{z} \sim 0.2$
}

\author{
L. Cortese ${ }^{1}$, D. Marcillac ${ }^{2}$, J. Richard ${ }^{3}$, H. Bravo-Alfaro ${ }^{4}$, \\ J.-P. Kneib ${ }^{5}$, G. Rieke ${ }^{2}$, G. Covone $^{6}$, E. Egami ${ }^{2}$, J. Rigby ${ }^{2}$, \\ O. Czoske ${ }^{7}$, and J. Davies ${ }^{1}$ \\ ${ }^{1}$ School of Physics and Astronomy, Cardiff University, Cardiff CF24 3AA, UK \\ ${ }^{2}$ Steward Observatory, University of Arizona, 933, N. Cherry Avenue, Tucson, AZ 85721, USA \\ ${ }^{3}$ Caltech, Astronomy 105-24, 91125 Pasadena CA, USA \\ ${ }^{4}$ Departamento de Astronomia, Universidad de Guanajuato, Mexico \\ ${ }^{5}$ Laboratoire d'Astrophysique de Marseille, Traverse du Siphon, BP8 13376 Marseille, France \\ ${ }^{6}$ INAF-Osservatorio Astronomico di Capodimonte, Napoli, Italy \\ ${ }^{7}$ Argelander-Institut für Astronomie, Universität Bonn, Bonn, Germany
}

\begin{abstract}
We report the discovery of two peculiar galaxies infalling into the lensing clusters of galaxies Abell $1689(\mathrm{z} \sim 0.18)$ and $2667(\mathrm{z} \sim 0.23)$. Hubble Space Telescope images show extraordinary trails composed by blue bright knots and stellar streams associated with both these systems, an $\sim L^{*}$ and $\sim 0.1 L^{*}$ galaxy. Under the combined action of tidal interaction with the cluster potential and of ram pressure by the intra-cluster medium the morphologies and star formation histories of these two galaxies are strongly perturbed. While in the massive system tidal interactions are the dominant effect and are able to produce a sinking of gas towards the galaxy center triggering a strong burst of star formation and changing galaxy's morphology, in the smaller galaxy the effects of gravitation are reduced by ram pressure stripping which blows away the neutral hydrogen from the galactic disk, quenching the star formation activity and transforming a gas rich late type spiral into quiescent disk dominated early type system. This result is a new additional evidence that galaxy mass represents the main driver of galaxy evolution, even during their dive into the harsh cluster environment and can give additional insights on the origin of S0s and dwarf cluster galaxies.
\end{abstract}

Keywords. galaxies:clusters:individual:(A1689,A2667)-galaxies:evolution-galaxies:interactions

\section{Environmental effect in Abell1689 \& Abell2667}

We carried out a multiwavelength analysis of two peculiar galaxies falling into the centers of the massive clusters Abell $2667(z \sim 0.23)$ and Abell $1689(z \sim 0.18)$. Because these two systems have significantly different optical luminosities $\left(\sim \mathrm{L}^{*}\right.$ and $\left.\sim 0.1 \mathrm{~L}^{*}\right)$ but are at similar distances from the cores of clusters of similar mass, they are ideal for a comparison of similar environmental effects on different-sized galaxies. Both these systems are associated with extended trails of bright blue knots and diffuse wisps and filaments of young stars. However while the galaxy in Abell2667 is experiencing a strong burst of star formation, appearing as a rare example of a luminous infrared cluster galaxy, the one in Abell1689 has recently ceased its star formation activity. The stripped blue knots have luminosities and physical sizes consistent with the ultra compact dwarf galaxies, suggesting that at least part of the population of ultra-compact dwarfs originate from young massive star clusters. 